\title{
Strategies for optimizing meso-scale spatial layout based on travel demand
}

\author{
Y. $\mathrm{Lu}^{1} \& \mathrm{X} . \mathrm{Zhu}{ }^{2}$ \\ ${ }^{1}$ Department of Architecture and Urban Planning, \\ Huazhong University of Science and Technology, China \\ ${ }^{2}$ Wuhan Planning and Design Institution, China
}

\begin{abstract}
Against the backdrop of the marketization of residential houses and the constant expansion of the scale of the city, traffic jams are becoming more serious, and the time consumed for travel by residents of large cities is prolonged. Areas of production are separated from the residential areas. Travel which used to take place within the community is forced to the use of city roads, and residents are facing serious traffic jam problems. So the reduction of motorized travel has become an important issue. This paper took travel demand as an important factor to measure the quality of the living environment and applied a questionnaire survey to analyse total travel, travel modes and travel frequency of residents. Firstly, taking Guanggu Block of Wuhan as a research case, this paper adopted a questionnaire survey method to analyse the quantity, type and frequency of block residents' travel and pointed out that $2 \mathrm{~km}$ is a critical distance, beyond which the number of journeys drops rapidly. Secondly, this paper compared the difference between ideal and practical travel frequency in different ranges of travel distance, showing that a considerable part of travel demand is not satisfied by land use in the meso-scale space. Thirdly, after identifying the current features of land use and the travel demand of Guanggu Block, this paper proposed that a functional mixture under a limited scale is an effective method to reduce the rate of motorized travel and unitize the meso-scale space. Moreover, in considering that much longdistance travel cannot be obviously reduced with mixed land use, it is necessary to arrange subway stations inside units of the meso-scale space. Finally, this paper proposed suggestions and strategies for optimizing the meso-scale spatial layout of large cities from the perspective of coordinating function and traffic.

Keywords: travel demand, land use, meso-scale space unit, non-motorized vehicle travel.
\end{abstract}




\section{Introduction}

At present, domestic research into the relationship between residents, travel behaviour and urban physical space focuses on two dimensions: macro scale at whole city level and micro scale at block level. The important point at the whole city level is to place high value on matching the city's living space with travel demand. Some scholars took district, county and street offices as spatial units to work out the statistics on commuting behaviour. Then, taking the commuting time of residents of each spatial unit and the ratio of the number of jobs to the number of residents, it showed a degree of matching between resident space and employment space [1-3]. Some scholars adopted a similar analysis method to interpret the existing problems of job-housing balance via analysis and evaluation of job-housing, matching the relation of each spatial unit according to the functions, area, and spatial structure determined in comprehensive planning $(\mathrm{Hu}$ et al. [4]). Research into micro scale focuses on the relation between the residents' commuting and the block's physical environment. Usually, blocks with typical characteristics are selected, based on dimensions like building year, land use and location [5], and relevance of the land use characteristics to non-motorized travel modes and distance of residents' travel are focused on. Moreover, it is generally believed that the commuting distance of residents is generally short, owing to the high degree of mixture of functions in an old town with mature development (Pan et al. [6]). It is commonly pointed out that the coupling of the functional mixture with the traffic structure and the functional space of the city can reduce commuting distance and car travel. However, research into the correlation of travel behaviour with city space shows that inadequate attention is paid to the meso-scale. In addition, past research has focused on a discussion of the relationship between composition of transport modes and characteristics of qualitative land use; research into the total quantity and frequency of journeys, which are closely related to residents' travel, is in short supply. As for the problem of how to structure a connection between the travel characteristics of residents with strategies for optimizing the meso-scale space, related studies have not discussed this matter at any depth.

\section{Study areas and methodology}

\subsection{Study area}

This paper surveyed residents of some regions of Guanshan Block of Hongshan District in Wuhan City, which are enclosed by four urban arterials in downtown Wuhan and adjacent to the sub-centre of Luxiang City, about $11.2 \mathrm{~km}$ away from the inner ring of Wuhan, covering an area of about $7.0 \mathrm{~km}^{2}$. The block's main functions are residential and retail industry; and most properties are commercial houses built after housing marketization reforms. It is therefore representative of a large city's meso-scale space. 


\subsection{Methodology}

The questionnaire divided residents' travel into three categories: travel of high frequency, medium frequency and low frequency, representing travel five times, three times and once a week, respectively. The questionnaire required every interviewee to fill in their travel of different frequencies and, for each, means of transport, purpose of travel, destination and time consumed for each journey. In order to ensure the reliability of the sample, the survey was conducted of residents who have lived in the region for more than half a year. Samples of the survey were checked at random, but two people at most per household were surveyed. Samples numbered 756, 614 of which were effective samples.

From residents' destinations, their distance of travel can be measured on a map. Meanwhile, according to their means of transport and travel time, their travel distance can be checked and finally, the distance of each journey can be calculated. Subsequently, range of travel distance was sub-divided: within a travel distance of between 0 and $10 \mathrm{~km}$, each $0.5 \mathrm{~km}$ is taken as a range of distance and journeys beyond $10 \mathrm{~km}$ are taken as another range of distance. Through such an operation, 21 ranges of travel distance were obtained (including $0 \mathrm{~km}-0.5 \mathrm{~km}, 0.5 \mathrm{~km}-$ $1.0 \mathrm{~km}, 1.0 \mathrm{~km}-1.5 \mathrm{~km} \ldots \ldots .9 .5 \mathrm{~km}-10 \mathrm{~km}$ and those beyond $10.0 \mathrm{~km})$. When the number of journeys is greater in one certain range, this means more active travel within that range. When the average frequency of travel is higher, it means that travel within that range of greater importance to the corresponding travellers (Fig. 1).

In a certain distance class, there are

a travels 5 times per week;

b travels 3 times per week;

c travels once per week.

The number of travels equals

$5^{*} a+3^{*} b+1{ }^{*} c$.

The average frequency equals

$\left(5^{*} a+3^{*} b+1^{*} c\right) /(a+b+c)$

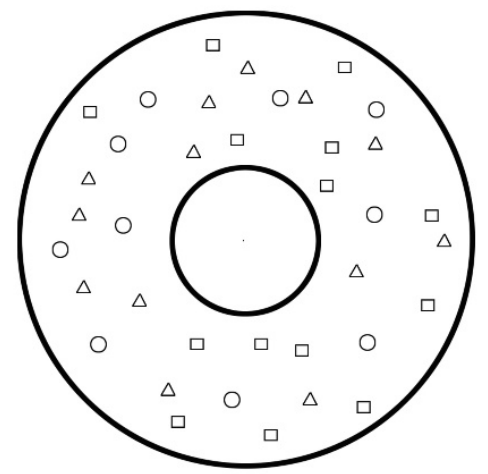

$\triangle 5$ times per week

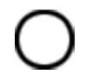

3 times per week

once per week

Figure 1: Travel amount and travel frequency calculation method in a certain distance class.

\section{Current travel demand}

\subsection{Travel distance}

After working out statistics on times of travel within each range of travel distance, the number of journeys occurring per week reached about 600-700 within the 0- 
$2 \mathrm{~km}$ travel distance range; these figures are obviously higher than the number of journeys within other ranges of distance. The numbering of journeys occurring per week within the range of distance beyond $2 \mathrm{~km}$ is obviously reduced. Within the range of $2-10 \mathrm{~km}$, the numbers of journeys in each range of distance are stabilized at 50-100 per week with the increase in travel distance (Fig. 2). These data point to $2 \mathrm{~km}$ being a critical scale to measure the active degree of travel. In general, journeys of less than $2 \mathrm{~km}$ are high in number, while outside this range, journeys in each range of travel distance are clearly reduced and fairly equally distributed across each range of distance.

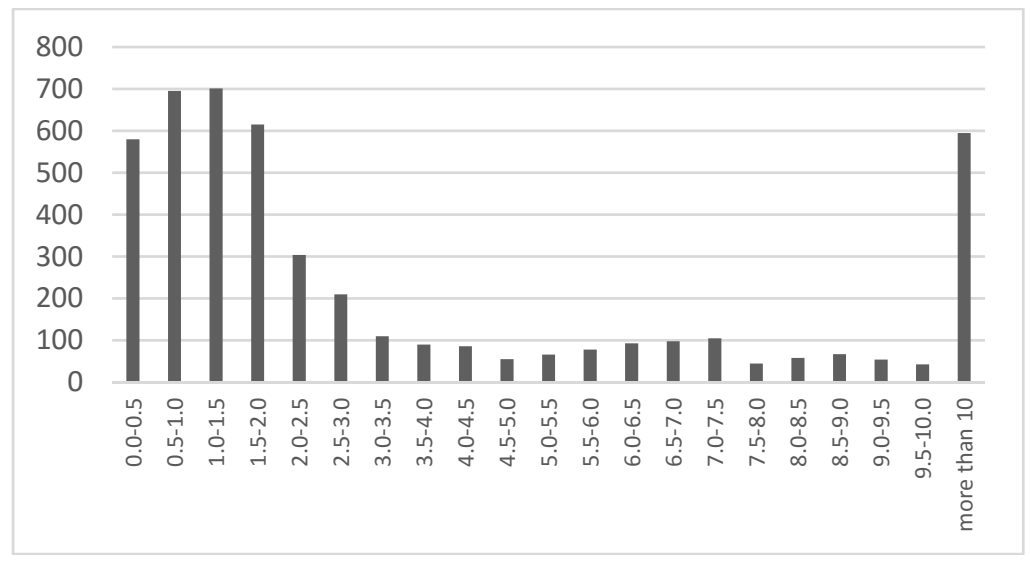

Figure 2: Travel amounts in different distance classes $(\mathrm{km})$.

\subsection{Travel purpose}

According to statistics, this research worked out the number of journeys for different travel purposes per week, for example going to work, school, shopping, participating in social communication, seeing the doctor, travelling for entertainment and leisure. The number of journeys for work and shopping are far greater than for travel of other types and make up $67.05 \%$ of the total (travel for work accounts for $40.93 \%$ and travel for shopping constitutes $26.12 \%$ ). The number of journeys for work is the greatest, about 1.6 times the number of journeys for shopping. So, journeys for work and shopping are the absolutely dominant status.

\subsubsection{Spatial distribution of travel for work}

Statistics found that, with an increase in travel distance, the number of journeys for work between each travel distance is generally reduced. About $25 \%$ of travel for work occurs within the scope of $1.0 \mathrm{~km}$. In the range of $1.0-3.5 \mathrm{~km}$ distance, the quantity of journeys for work is clearly reduced with the increase in travel distance. In the range of 3.5-10.0 km distance, the numbers of journeys for work in each section of distance are maintained at a low level. The distance of about $18 \%$ of journeys for work is more than $10.0 \mathrm{~km}$ (Fig. 3). These data illustrated that, 
although travel distance is an important factor influencing selection of work place and living place, the distance of travel for work for quite a considerable number of residents is long, exceeding the $2 \mathrm{~km}$ critical scale for measuring active degree of travel.

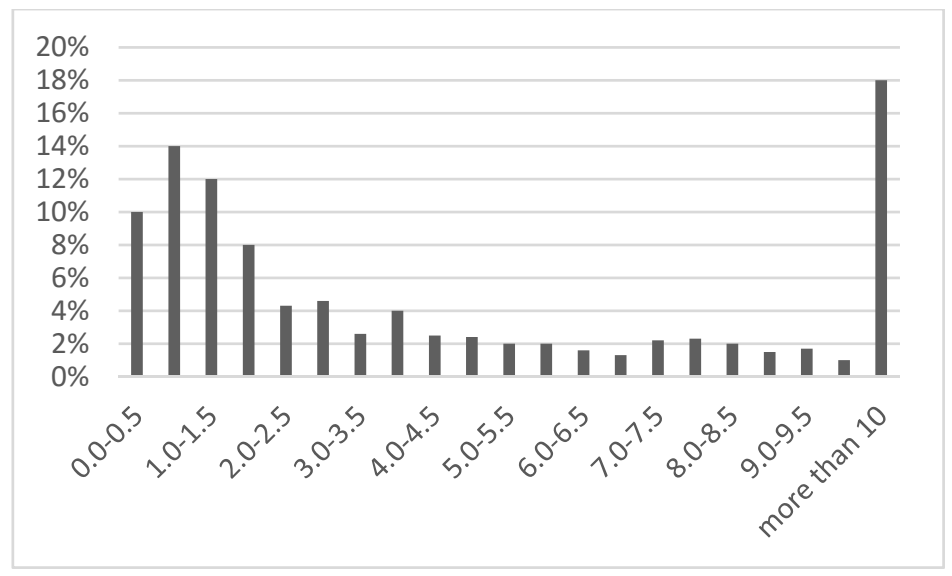

Figure 3: Work commuting travel ratios in different distance classes $(\mathrm{km})$.

\subsubsection{Spatial distribution of travel for shopping}

Statistics found the degree of sensitivity to travel distance is far higher in journeys for shopping than in travel for work. Of journeys undertaken for shopping, 56.9\% occur within the range of $0.5 \mathrm{~km}$. Travel for shopping within the scope of $2 \mathrm{~km}$ accounted for $86.2 \%$ of total shopping travel. In ranges of distance beyond $2 \mathrm{~km}$, the rate of travel for shopping is not more than $5 \%$ (Fig. 4). These data showed that, in their travel for shopping, most residents will not go to shop in places at a distance of more than $2 \mathrm{~km}$, and the daily shopping needs of people can be basically satisfied within a spatial scope of $2 \mathrm{~km}$.

\subsection{Features of travel frequency}

The research worked out statistics for the average value of frequency of travel in each range. When the value is higher, it means a higher frequency of travel in that range of distance, in general. Statistics showed that the average value of travel frequency reached peaks in the $3.5-4.0 \mathrm{~km}$ range and the $8.5-9.0 \mathrm{~km}$ range, at 3.95 times and 4.00 times per week, respectively (Fig. 5). The average value of travel frequency in other ranges of distance is basically between 3.0 and 3.5. In further statistics on the distribution of travel within the 3.5-4.0 km range and the 8.5$9.0 \mathrm{~km}$ range, travel of high frequency was found to reach $90.4 \%$ and $85.9 \%$, respectively, of the total amount of travel in the two ranges of distance, with travel for work taking the majority, reaching $54.2 \%$ and $70.3 \%$, respectively. These data indicated: journeys of more than $2 \mathrm{~km}$ distance are quite important for a section of residents and are mainly undertaken for work. Therefore, long-distance travel for work is an indispensable part of the travel routine of residents. 


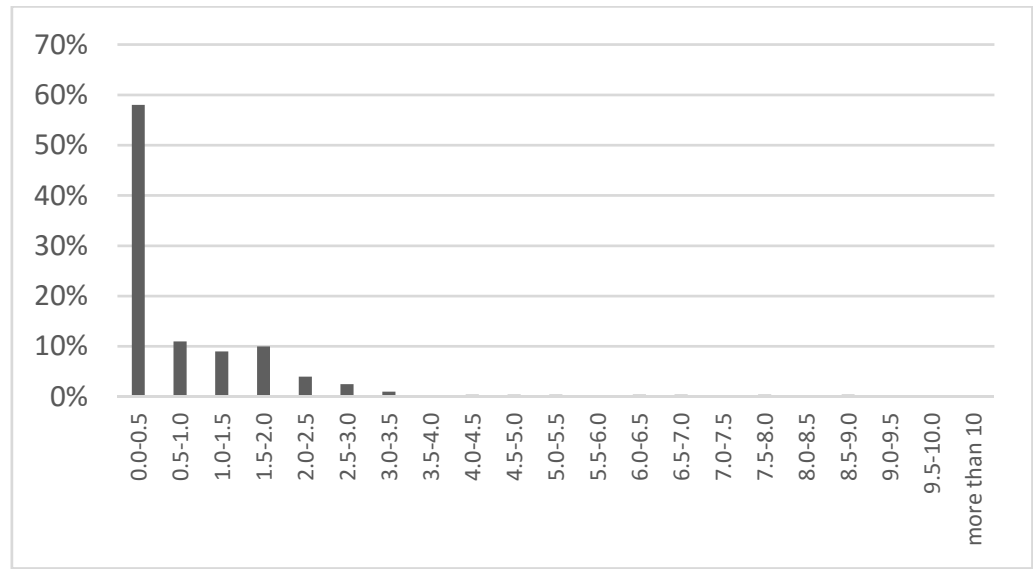

Figure 4: $\quad$ Shopping travel ratios in different distance classes $(\mathrm{km})$.

\section{Spatial characteristics demanded by residents from the perspective of reducing motorized travel}

\subsection{Features and problems in current travel demand}

\subsubsection{Short-distance non-motorized travel}

Non-motorized travel includes travel on foot and travel with bikes. The tolerance level of non-motorized travel is lower than that of motorized travel; this is determined by the features of different transportation media. Through research on the correlation of travel modes and travel distances of residents of six Chinese cities, Chen et al. [7] found that a high number of residents travel on foot within the travel scope of $0-3 \mathrm{~km}$, while, when the travel distance exceeds $3 \mathrm{~km}$, the rate of travel on foot is sharply reduced to lower than $30 \%$. Via a survey on the journeys of residents of Beijing, Chang [8] worked out statistics on the rate of each travel mode inside each short-distance range, finding that travel on foot and by bike makes up the majority within a distance of $0-2 \mathrm{~km}$. This is illustrated by the fact that non-motorized travel takes up $95 \%$ within the $0-1 \mathrm{~km}$ travel distance and $81 \%$ within the $1-2 \mathrm{~km}$ travel distance.

Therefore, journeys on the $0-2 \mathrm{~km}$ scale, which constitute the majority of residents' travel, as revealed in the survey, can be basically undertaken without the need for motorized travel. In general, the distance of most residents' travel is short, and travel within the non-motorized travel scale is active.

\subsubsection{Frequency of long-distance travel}

In order to find problems in the current travel demand of residents, it is necessary firstly to identify the travel demand of residents under ideal state conditions. As obstructions to travel increase with the increase in travel distance, the quantity and frequency of residents' journeys should be reduced with an increase in travel distance. From the perspective of the ideal travel demand of residents, short- 
distance travel should be more frequent than long-distance travel . For example, in order to save travel time, white collar workers who need to commute every working day usually choose to live near their workplace and routine travel for buying vegetables and entertainment is easily satisfied nearby. When travel, which is only necessary once a month or once a year, is conducted (such as going to downtown to buy high-end commodities), they will accept long-distance travel. However, the survey found that, although the number of journeys basically conforms to the rule, data on the frequency of travel does not conform to it. Some long-distance journeys are too frequent, while short-distance travel is not frequent enough.

\subsection{Disadvantageous influence of current spatial layout on travel demand of residents}

The above analysis found some long-distance travel occurs too frequently, but the frequency of travel within the $0-2 \mathrm{~km}$ range is too low. Oriented by non-motorized travel, the increasing frequency of travel within the scale will further increase the rate of non-motorized travel. The too high frequency of some long-distance travel is attributed, to a great extent, to too many long-distance journeys of high frequency for work. As city space cannot meet this part of travel demand for work, it is necessary for some travel for work to be solved within a bigger scope.

Thus, it seems that the unreasonable alienation of cities' spatial layout distorts the travel of residents. City functions within a range of $0-2 \mathrm{~km}$ away from residential places are of insufficient diversity, which forces some residents to conduct long-distance travel at high frequency.

\subsection{Main points for spatial layout of meso-scale}

\subsubsection{Layout of functional mixtures within a limited scale}

From the perspective of non-motorized travel, the situation of whether the layout of a meso-scale space can satisfactorily meet the travel demand of residents is determined by whether the non-motorized travel scale contains functions necessary for the daily life of residents, as it is an important point, which influences the travel demand of residents. So, the meso-scale space should further strengthen the uniform functional mixture within the range of $2 \mathrm{~km}$. With Guanggu Block as an example, its functional mixture is still mainly existent at the level of the general scale. Though cities' diversified functions exist inside the meso-scale space of about $7 \mathrm{~km}^{2}$, the feature is not obvious inside a relatively small-scale space, as the same type of land use forms large and continuous patches, and the whole block consists of several homogeneous patches. Future spatial layout should focus more attention on a uniform functional mixture and improve functional allocation within the range of the $0-2 \mathrm{~km}$ scale. Strengthening the active degree of travel within $0-2 \mathrm{~km}$ will encourage residents to adopt more non-motorized travel.

\subsubsection{Non-motorized accessibility of subway stations}

From the survey, we learn that quite a lot of travel for work is long distance, indicating that long-distance travel is not a rigid restrictive factor for residents in 
selecting their workplace. On the contrary, travel itself for work is a more rigid factor. The issue of whether there is an employment position around the living zone is not necessarily connected with whether residents select employment nearby. Against the background of housing marketization, the demand for longdistance travel for work it cannot completely be removed, even if a functional mixture is constructed within a limited scale.

In order to relieve traffic jams on the traffic arteries of cities, long-distance travel should be transferred into a combination of short-distance travel of the meso-scale with rail transit. So, non-motorized accessibility to large-capacity public transport stations is an inevitable requirement in order to reduce private long-distance travel when there is a strong demand by residents for long-distance travel for work. However, when the road system of one block lacks an integrated branch system, residents still need to strongly depend on main and secondary arteries for their access to rail transit stations. In fact, poor non-motorized accessibility to rail transit stations may cause residents of the block with a need for long-distance travel to adopt private motorized methods.

\section{Suggestions and strategies for optimizing spatial layout of meso-scale}

\subsection{Unitize meso-scale space within a limited scale}

\subsubsection{Functional composition}

A functional mixture within a limited scale scope not only aims to guarantee local travel for residents within the meso-scale space, but it also tries to structure relatively independent meso-scale space units Through a functional mixture within a limited scale, such specific meso-scale space units with a clear boundary can meet the travel demand of residents inside and reduce long-distance travel, which steps over the meso-scale space unit. Under unit-based meso-scale space organization, a part of the travel demand, which needs to be satisfied with longdistance travel, will be shifted to the meso-scale space unit.

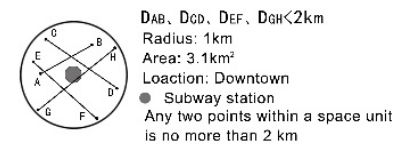

Urban space unit spatial structure $A\left(3.1 \mathrm{~km}^{2}\right)$

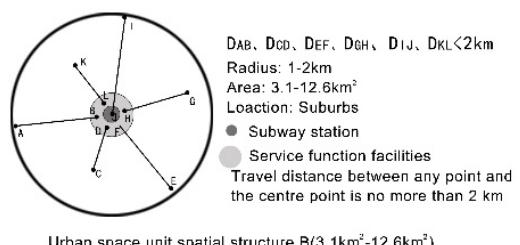

Urban space unit spatial structure $B\left(3.1 \mathrm{~km}^{2}-12.6 \mathrm{~km}^{2}\right)$

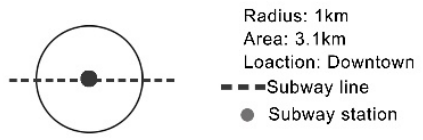

Urban space unit spatial structure $A\left(3.1 \mathrm{~km}^{\prime}\right)$

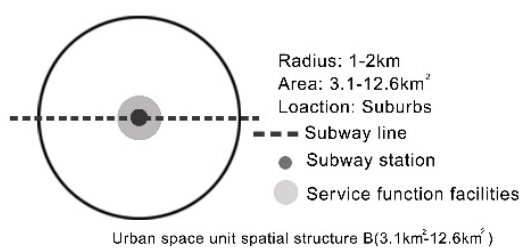

Figure 5: Spatial structure of meso-scale urban space unit in different scales (left: function model; right: traffic model). 
The critical value of $2 \mathrm{~km}$ to measure the active degree of travel obtained from the survey can be taken as a basis of a functional mixture within an acceptable scope of non-motorized travel modes. As a scale threshold of $2 \mathrm{~km}$ can be extended into two scale values of a meso-scale space unit: (1) When the travel distance between any two points within one space unit is within the scope of $0-2 \mathrm{~km}$, a circular zone with a diameter of $2 \mathrm{~km}$ can be worked out, covering an area of about $3.1 \mathrm{~km}^{2}$. So, all travel can be controlled within the range of $0-2 \mathrm{~km}$ if various functions are part of a mixed layout in the zone. (2) When the distance of any point of one space unit to its centre is within the range of $0-2 \mathrm{~km}$, a circular zone with diameter of $4 \mathrm{~km}$ will be worked out, covering an area of $12.6 \mathrm{~km}^{2}$.

The mixture inside the space unit refers to a kind of limited mixture of spatial layout or the structural centre of the unit must have a concentrated layout of various functional facilities to ensure that the distance of all residents of the unit from the various functional facilities is within the scope of a $0-2 \mathrm{~km}$ scale (Fig. 6). So, the scale of the meso-scale space unit should be between $3.1 \mathrm{~km}^{2}$ and $12.6 \mathrm{~km}^{2}$.

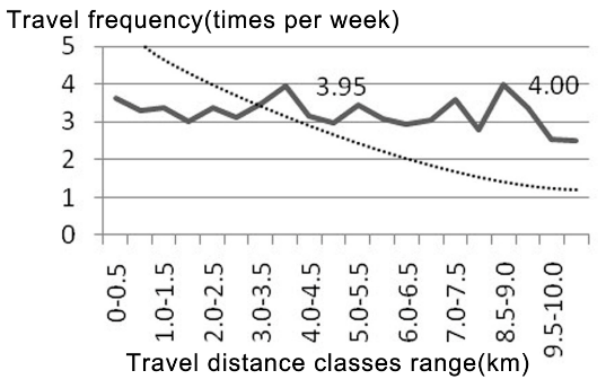

Figure 6: Comparison of current travel frequency curve in Guanggu district and ideal travel frequency curve (dashed line).

\subsubsection{Traffic organization}

The above mentioned survey found quite a lot of high-frequency long-distance commuting travel. Based on the practical functional layout of cities at present, a long process is needed to guide the mixture of functions and it is not practical to balance job/housing within the short term via a mixed layout of functions within a limited scale. So, the demand for high-frequency long-distance travel needs to be effectively satisfied with the aid of rail transit.

A meso-scale space structure oriented by rail transit is a "cell" structure with a rail transit station at the core, aiming to ensure that the areas around the station can be serviced equally, which means that all residents can have equal access to the station. Targeted at a space unit with an area between $3.1 \mathrm{~km}^{2}$ and $12.6 \mathrm{~km}^{2}$, its traffic organization is mainly composed of two parts: part 1 refers to traffic inside the unit, or to reach a functional place necessary for daily life within the range of $2 \mathrm{~km}$ travel distance; part 2 is concerned with long-distance travel with the aid of rail transit, and people can reach rail transit stations located at the core from any point of the unit. The layout of public service facilities shall be relatively 
concentrated in a combination of the structural centre of the space unit with rail transit stations, and the density of branch roads should be enhanced so as to promote the accessibility of rail transit stations (Fig. 6).

\subsection{Space docking of meso-scale space unit and subway network}

The scale of the meso-scale unit differs at different locations of one city. As the number of various service facilities needing to be supported by a certain base of the population differs in different locations the meso-scale unit of the suburbs needs to have a sufficient base number of population to support various service facilities within a large space. In addition, the passenger capacity of each rail transit station is basically equal, so, in order to ensure the efficiency of public transport, the interiors of suburban stations must be bigger than that of downtown. In the centre of large cities, a base number of the population is needed to support various service facilities; this can be achieved within a relatively small scope of the region owing to the high density of population. In that way, functional organizations in downtown can meet the routine travel demands of residents within a small scope of scale. So, a small-scale unit space $\left(3.1 \mathrm{~km}^{2}\right)$ within a diameter of $1 \mathrm{~km}$ can be situated at the city centre. With the increasing distance to the city centre, the area of the space unit will be increased gradually, but the maximum area of the space unit shall not exceed the one $\left(12.6 \mathrm{~km}^{2}\right)$ with a diameter of $4 \mathrm{~km}$ (Fig. 7).

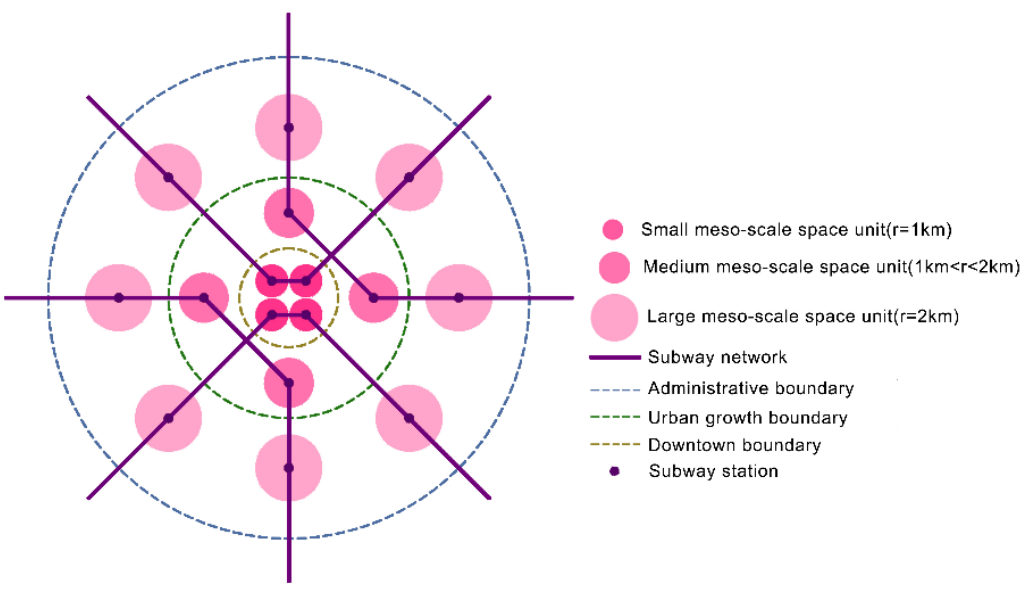

Figure 7: Meso-scale urban space unit layout connecting with metro network.

\section{Conclusion}

With Guanggu Block of Wuhan as a typical large-city meso-scale space, this paper proposed a "cell" model of a meso-scale space unit and strategies for optimizing land use to reduce the demand for motorized travel. 
Analysis results showed that the active scope of residents' travel activities is about $2 \mathrm{~km}$. In order to effectively meet the travel demands of residents, various functions, which are closely related to the routine journeys of residents, shall be mixed within a limited scope of scale to solve more routine travels within a specific meso-scale space and form a relatively perfect "unit-based" meso-scale space for city functions. Secondly, while confronted with the long-distance rigid demand for high-frequency travel to work, the meso-scale space must be combined with rail transit stations; the accessibility of internal branch roads must be improved in order to strengthen non-motorized accessibility between rail transit stations and living areas.

For Guanggu Block, large-scale business development over the last 10 years can meet the travel demands of residents for shopping, but the considerable, strong demand for travel for work is in contradiction to the insufficient coverage of largecapacity rail transit. The lack of an internal branch road system within the block weakens the non-motorized accessibility of rail transit stations, which further enlarges the contradiction, thus making it easy to induce new long-distance private motorized travel. The functional organization and spatial layout of the meso-scale space are comprehensively influenced by numerous factors. However, this analysis of the travel demand of residents is conducted based on one dimension alone, leaving many problems requiring further study.

\section{Acknowledgement}

Project aided by the National Science Foundation of China (No. 51478199; 51538004).

\section{References}

[1] Meng Bin, The spatial organization of the separation between jobs and residential locations in Beijing. Acta Geographica Sinica, 64(12), pp. 14571466, 2009.

[2] Zhao Hui; Yang Kaizhong; Wei Haitao; Zhao Wenyi, Job-housing space restructuring and evolution of commuting patterns in Beijing metropolitan area. City Planning Review, 37(8), pp. 33-39, 2013.

[3] Qian Linbo, The research on the relationship between degree of mixed urban land-use and spatial distribution of trips: In case study of main districts in Nanjing. Modern City Research, 7(3), pp. 7-10, 2000.

[4] Hu Juan; Hu Yidong; Zhu Lixia, Exploration on spatial development of Wuhan based on job-housing balance. City Planning Review, 37(8), pp. 2532, 2013.

[5] Zhou Suhong; Yan Xiaopei, The impact of commuters' travel pattern on urban structure: a case study in some typical communities in Guangzhou. Acta Geographica Sinica, 61(2), pp. 179-189, 2006.

[6] Pan Haixiao; Liu Xianteng; John Zacharias; Liu Bing; Liao Xiongjiu, Neighbourhood design characteristics and the choice of green transport. Urban Planning Forum, 10(6), pp. 42-48, 2003. 
128 The Sustainable City XI

[7] Chen Zheng, Liu Ying-shun, Shi Fei, Study on distance division curve of inhabitant trips modal. Communications Standardization, 26(8), pp. 157-159, 2006.

[8] Chang Chaofan, Study on the urban residents short-distance travel behaviour. 1 December 2007, Beijing Jiaotong University, Beijing, China, 2007. 